increase in TBR for the non-ADHD groups. A substantial sub-group of ADHD patients do deviate on the TBR measure, but excessive TBR is not a reliable diagnostic measure of ADHD. It may have prognostic value. (Arns M, et al. A decade of EEG theta/beta ratio research in ADHD: a meta-analysis. J Atten Disord 2013 Jul;17(5):374-83).

COMMENTARY. The FDA approved the Neuropsychiatric EEG-Based ADHD Assessment Aid (NEBA) medical device in 2013 to be used as confirmatory support or to pursue further testing after an evaluation for ADHD, in a child aged 6-17. The device was not to be used as a stand alone method of diagnosis of ADHD.

The AAN, in an Evidence-Based Practice Advisory, concludes that it is highly likely that EEG theta-beta power ratio and EEG frontal beta power correctly identify patients with ADHD (accuracy $89 \%$ to $94 \%$ ) as compared to a clinical evaluation. The AAN recommends that the EEG test should not be used in place of a standard clinical evaluation, because of the risks of misdiagnosis of $6-15 \%$ when using the theta/beta ratio. There is neither evidence for, nor against the use of theta/beta EEG power ratio either to confirm a diagnosis of ADHD, nor to support further testing. Whether comorbid disorders such as ODD have similar changes in the theta/beta ratios that mimic the reported finding in ADHD is not known [1].

A recent report of spectral analysis of EEGs on 28 normal and 58 ADHD children, aged 6 to 14 years, found TBR was higher in ADHD subjects, with lower beta but no difference in theta power over Broca's area. Beta-1 power over Broca's area was the best diagnostic test, with sensitivity 0.86 and specificity 0.57 . The EEG beta-1 power and TBR assist in confirming the diagnosis of ADHD in a sample with moderate pretest probability of ADHD [2].

The present symptomatic method of diagnosis, based on parent and teacher evaluations, is relatively accurate in children with the hyperactive-impulsive subtype of ADHD but less so with the inattentive type. A more objective test such as EEG if validated could be a valuable aid in the diagnosis and management of ADHD. The significance of seizure discharges in approximately $25 \%$ of sleep-deprived EEGs in ADHD children is further evidence of the utility of the EEG in ADHD management [3].

\title{
References.
}

1. AAN. Clinical Practice Guideline Process Manual. 2011 Ed. St Paul, MN: Am. Acad. of Neurology.

2. Sangal RB, Sangal JM. Clin EEG Neurosci. 2014 Jun 26. [Epub ahead of print].

3. Millichap JJ, Stack CV, Millichap JG. J Child Neurol. 2011 Jan;26(1):6-11.

\section{TREATMENT OF ADHD}

\section{EFFECT OF METHYLPHENIDATE ON INATTENTION DURING DRIVING}

Investigators at Utrecht University, the Netherlands; and centers in Australia and Detroit, MI, evaluated the lapses of attention during on-road highway driving in 18 adult ADHD patients during treatment with methylphenidate (MPH) or placebo. Driving was significantly better with MPH when compared with placebo, with reduction in weaving, lapses, and inattention. Lapses were common on placebo (11/18 patients), and much less after MPH (5/18). Lapses often go unnoticed by drivers. (Verster JC, Roth T. 
Methylphenidate significantly reduces lapses of attention during on-road highway driving in patients with ADHD. J Clin Psychopharmacol 2014 Jun 27. [Epub ahead of print]).

COMMENTARY. ADHD young adults are twice as likely to be cited for unlawful speeding and have more crashes and more accidents involving bodily injury, when compared to non-ADHD adult control subjects. These findings support a need for continued treatment of ADHD into adolescence and adulthood. Improvement of driving performance of adolescent drivers with ADHD was demonstrated using a driving simulator while taking Concerta compared to placebo [1] or immediate-release MPH [2].

References.

1. Cox DJ, et al. Pediatrics. 2006 Sep;118(3):e704-10.

2. Cox DJ, et al. J Am Acad Child Adolesc Psychiatry. 2004 Mar;43(3):269-75.

\section{GUANFACINE EXTENDED RELEASE IN ADHD}

Investigators at Massachusetts General Hospital, Boston, MA, performed a multicenter, 9-week, double-blind, placebo-controlled, dose-optimization study of guanfacine extended release $(\mathrm{GXR},<4 \mathrm{mg} / \mathrm{d})$ adjunctive to a long-acting psychostimulant for ADHD continued in 461 subjects. Patients were randomized to receive GXR in the morning (GXR AM), GXR in the evening (GXR PM), or placebo.

GXR treatment groups showed significantly greater improvement from baseline compared with placebo plus psychostimulant. Small mean decreases in pulse, systolic, and diastolic blood pressure were observed in GXR treatment groups. Morning or evening GXR administered adjunctively to a psychostimulant showed significantly greater improvement and generated no new safety signals. (Wilens TE, et al. A controlled trial of extended-release guanfacine and psychostimulants for attentiondeficit/hyperactivity disorder. J Am Acad Child Adolesc Psychiatry 2012;51(1):74-85).

COMMENTARY. A similar randomized, double-blind trial of guanfacine extended release (Intuniv) administered either in the morning $((\mathrm{n}=107)$ or evening $(\mathrm{n}=$ 114) was associated with significant improvements in ADHD symptoms. Once-daily GXR monotherapy is effective, administered AM or PM [1]. The rapid-release guanfacine (Tenex) is often prescribed as an alternative to Intuniv because of cost and insurance denial. Tenex is often effective in the hyperactive/ADHD younger child with sleep disorder or tics, but drowsiness during the day may lessen the ability to focus in school. The effect of Intuniv on school performance and grades requires further study.

\section{References.}

1. Newcorn JH, et al. J Am Acad Child Adolesc Psychiatry. 2013 Sep;52(9):921-30.

\section{ADVERSE EFFECTS OF MEDICATIONS FOR ADHD}

\section{CARDIAC AUTONOMIC DYSFUNCTION AND STIMULANT THERAPY}

Investigators at University of Minnesota, Minneapolis, report cardiac autonomic dysfunction and arterial stiffness among children and adolescents with ADHD treated 